\title{
MODIFICAÇĀO DO METODO "KINDLING" PARA OBTENÇAO DE STATUS EPILEPTICUS EXPERIMENTAL EM RATOS
}

\author{
CARLOS J. REIS DE CAMPOS* \\ ESPER A. CAVALHEIRO **
}

Goddard (1967) e Goddard, McIntyre e Leech (1969), mediante estimulação elétrica de cérebros de ratos, gatos e macacos observaram que a primeira estimulação tinha pouco efeito sobre o comportamento e não causava pósdescargas eletrográficas e que a resposta à estimulação se modificava progressivamente com a repetição, induzindo descargas epilépticas, automatismos comportamentais e, eventualmente, convulsões clônicas bilaterais. Essas alterações progressivas que resultavam da estimulação elétrica repetitiva foi por eles denominada de "kindling effect". O número de estimulações necessárias para a obtenção de crises motoras induzidas por "kindling" foi inversamente relacionado à duração dos intervalos inter-estímulos. Foi difícil, se não impossível, obter "kindling" em ratos, com intervalos inter-estímulos menores que $20 \mathrm{mi}$ nutos, sendo que intervalos de 24 horas ou maiores foram considerados ótimos. Assim, segundo Goddard e col. (1969), o número de estímulos necessários para a obtenção do "kindling", com crises motoras, diminui à medida que o intervalo entre os estímulos se aproxima de 24 horas.

A preparação "kindling" tem definida vantagem sobre outras preparações que envolvem a aplicação cerebral direta de irritantes químicos como o hidróxido de alumínio, porque o "kindling" oferece oportunidade para se investigar uma grande quantidade de parâmetros associados com um padrão altamente predizível de desenvolvimento de crises e um local anatômico de estimulação facilmente identificável (Goddard e col, 1969; Wada e Sato, 1974).

Taber, McNamara e Zornetzer (1977), mediante estimulação elétrica intermitente hipocampal dorsal, um estímulo por minuto, em camundongos, obtiveram um estado duradouro de crises convulsivas eletrográficas e comportamentais que foi considerado como um modelo de status epilepticus experimental.

Neste nosso trabalho procuramos confirmar os resultados destes últimos autores, em uma outra espécie animal (ratos), já que a modificação por ele introduzida permite a obtenção do fenômeno "kindling" em tempo bem mais curto (horas), comparativamente às técnicas anteriormente descritas (dias).

Trabalho da Disciplina de Neurofisiologia do Departamento de Fisiologia, da Escola Paulista de Medicina, realizado com o auxilio financeiro da FAPESP, do CNPq e da FINEP: * Bolsista do CNPq; Chefe da Disciplina de Neurofisiologia. 


\section{MATERIAL E METODO}

Foram utilizados 12 ratos machos albinos, raça Wistar, com aproximadamente 120 dias de idade, pesanjo entre 220 e $250 \mathrm{mg}$ no inicio da experiencia, provenientes do Biotério Central da Escola Paulista de Medicina. Todas as preparações animais utilizadas foram crônicas. Os animais foram operados sob anestesia com cloridrato de ketamina (60 mg/kg.IM) e pentobarbital (20 $\mathrm{mg} / \mathrm{kg}$.IP).

Foram implantados esterestaxicamente eletrodos de aço inoxidável com 100 micrômetros de diametro, duplos e torcidos, no hipocampo dorsal esquerdo (segundo o atlas de De Groot: antero-posterior $=3,0 \mathrm{~mm}$; lateral $=3,0 \mathrm{~mm}$ e vertical $=2,8 \mathrm{~mm}$, a partir da superfície cortical). Foram, também, colocados eletrodos neocorticais epidurais bilateralmente e um eletrodo subcutaneo utilizado como "indiferente".

Os registros da atividade elétrica hipocampal e neocortical foram feitos, num poligrafo Beckman tipo "RM dynograph" de oito canais. A amplificação do sistema era escolhida de acordo com a voltagem de cada derivação no momento do registro. A velocidade do papel era mantida, habitualmente em $10 \mathrm{~mm} /$ segundo.

A estimulação elétrica hipocampal foi realizada mediante um estimulador de ondas quadradas, bifásicas de $60 \mathrm{~Hz}$ que emite estímulos intermitentes durante 1 segundo cada, com uma intensidade que variava de 300-800 $\mu$ A. Foi utilizado um "switch-box" que permitia estimulação intermitente com intervalo constante, isto é, 1 segundo de estímulo seguido por 59 segundos de registro da atividade elétrica. A estimulação prosseguiu durante 120 minutos em todas as sessões.

A avaliação da atividade epiléptica eletrográfica foi feita considerando-se o tempo cle duração de padrões epilépticos eletrográficos como, por exemplo, espículas, poliespiculas. espícula-onda. expresso em número de segundos de atividade epiléptica por minuto de registro.

\section{RESULTADOS}

Vários padrões de pós-descargas puderam ser observados durante os experimentos (Fig. 1). Não foi possível qualquer correlação entre a atividade elétrica registrada e o comportamento do animal, exceto quan lo este apresentava "sacudidelas corporais" (do inglês, wet-dog shaikes). Nestes periodos observou-se, no traçado, surtos de poliespiculas intercalados com depressão da amplitude do registro (Fig. 2 - B e C). O comportamento dos animais variou desde a ausencia de qualquer movimento observável até manifestaçóes motoras estereotipadas com movimentos mastigatórios rítmicos, clônus de uma ou mais patas, sacu didelas corporais repetidas, com quedas intermitentes ou nåo.

Na figura 3, observa-se a generalização do fenômeno das pós-descargas para além do local estimulado (isto $e$, hipocampo dorsal esquerdo), as quais foram também registradas no hipocampo contralateral e nas regiões neocorticais bilaterais.

Na figura 4 observa-se uma pós-descarga hipocampal e neocortical típica seguida de uma depressão da amplitude do registro bastante evidente no hipocampo. Essa depressåo não foi observada constantemente após todas as pós-descargas. 

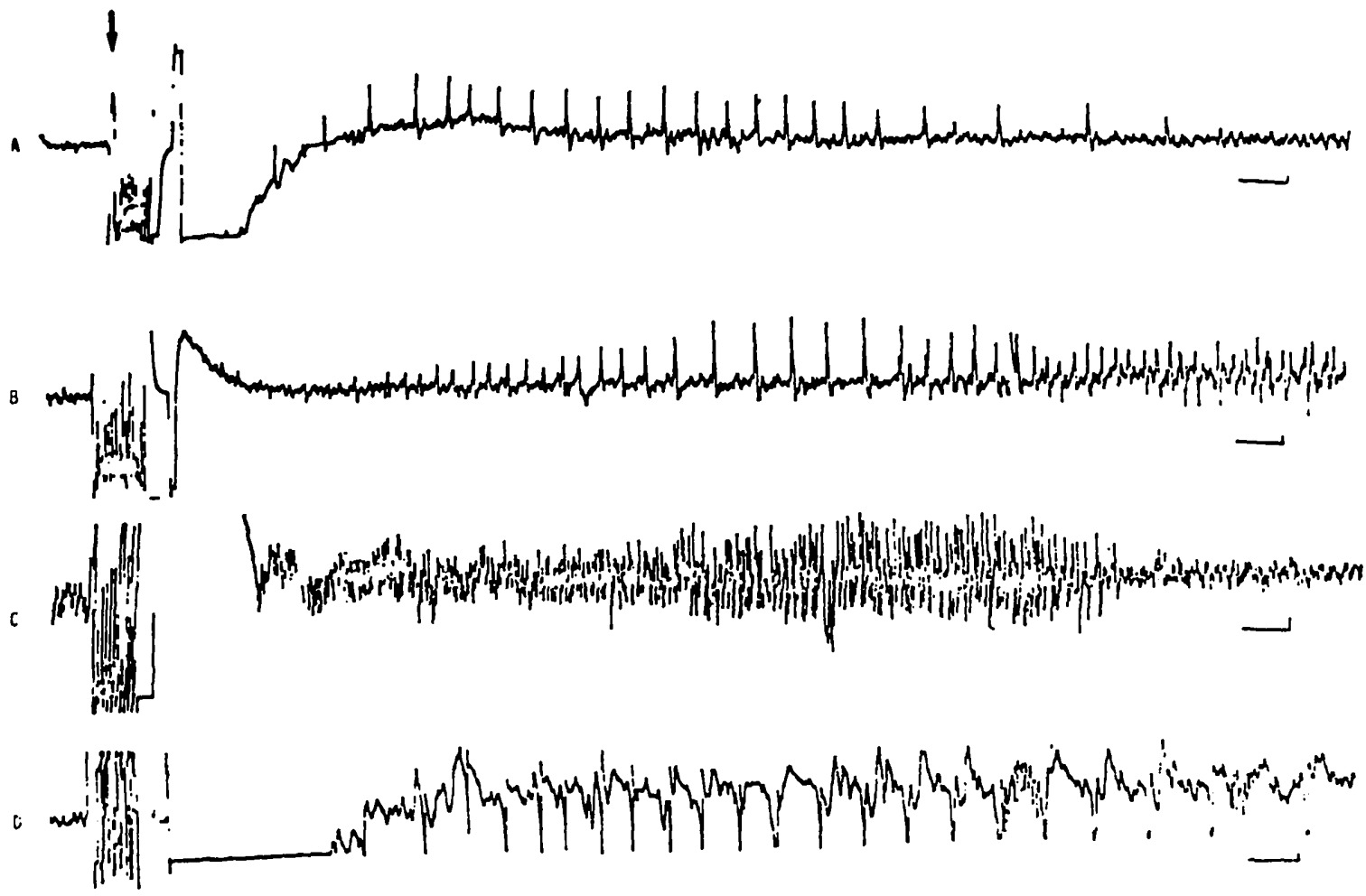

Fig. 1 - Padroes de pos-descargas observados: no tracado $A$, ap $\delta$ s o artefato de estimulo (seta) observa-se o aparecimento de descargas epilépticas, tipo "ponta", aproximadamente 1 por segundo; tracado $B$, espiculas com amplitude crescente e frequencia varidvel; tracado $C$, espiculas em maior frequencia (aproximadamerate 10/seg); tracado $D$, atividade epiléptica hipocampal tipo "ponta-onda" (aproximadamente s/seg). Calibraçao $=50 \mu^{V / 1}$ seg.

$\boldsymbol{\Lambda}$
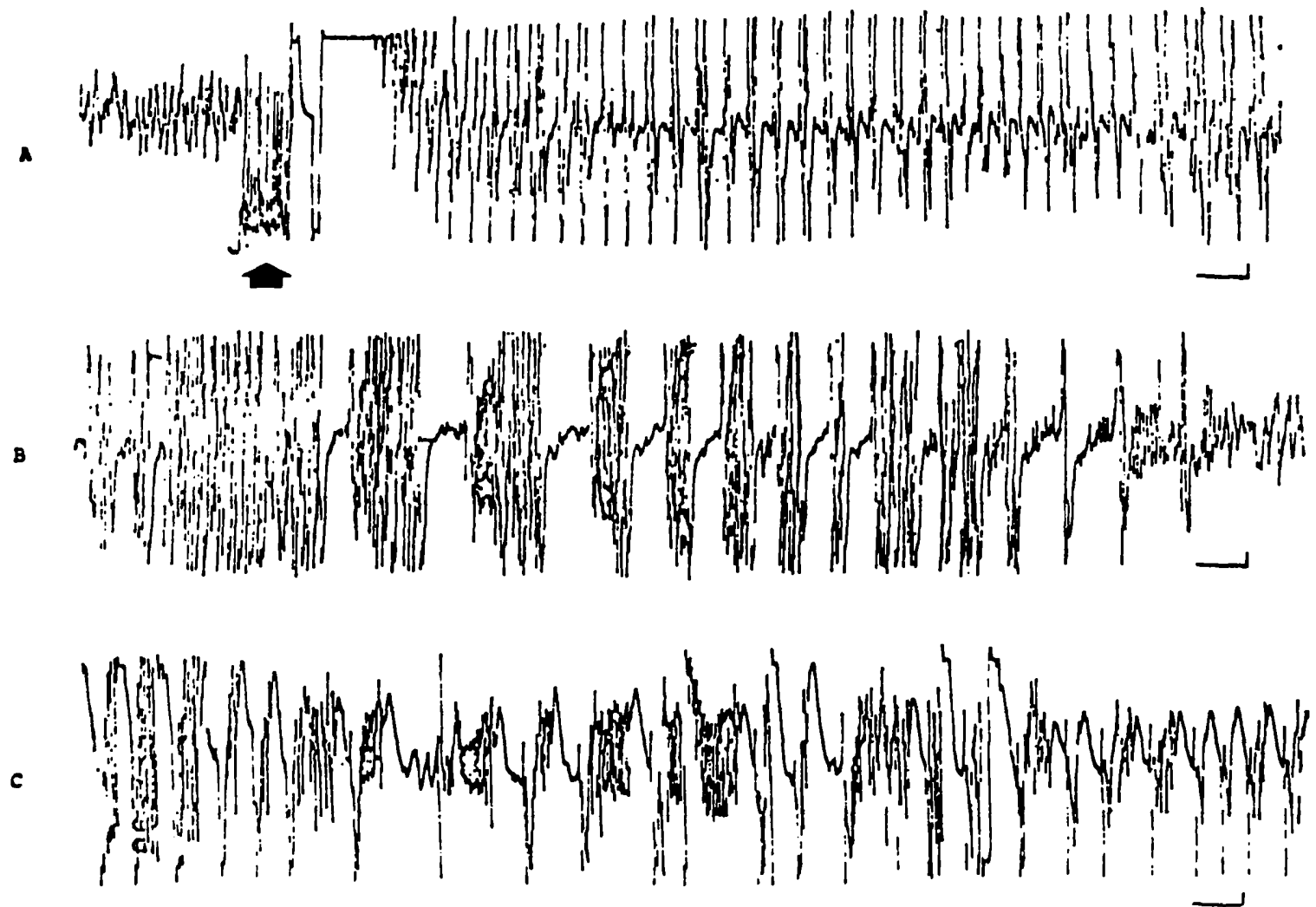

Fig. 2 - Tracado $A$, após o artefato de estimulo (seta) observa-se um padrāo comum de pos-descarga. Tracados $B$ e $C$, surtos de poliespiculas intercalados com depressdo da amplitude do registro. Nessa ocasido - animal apresentava "sacudidelas corporais". Calibragdo $=50$ $\mu V / 1$ seg. 


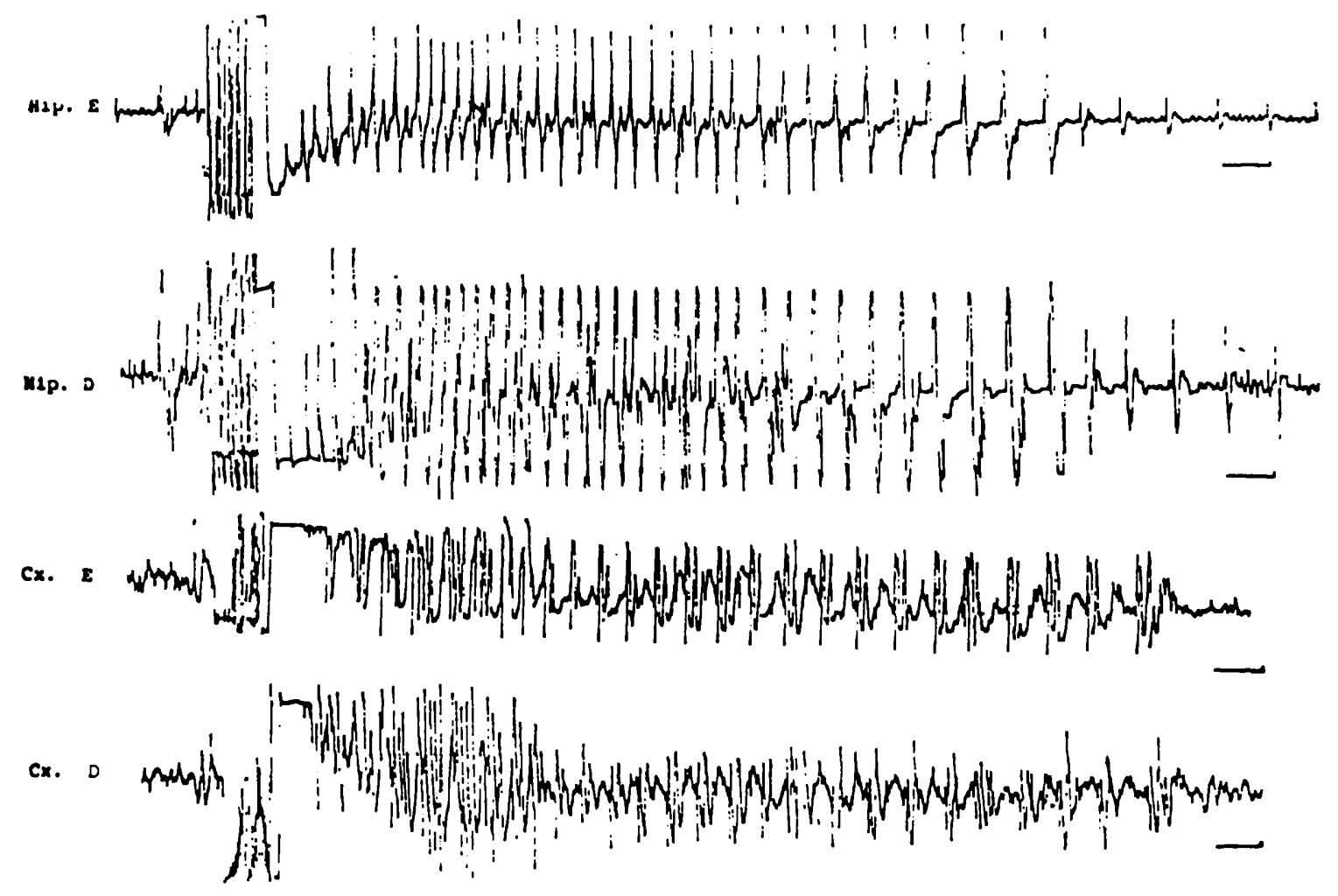

Fig. 3- Observa-se a generalização do fenómeno das pós-descargas para além do local estimulado (hipocampo E); Hip = hipocampo; $C x=$ neocórtex. Calibracão $=50{ }_{\mu} \nabla / 1$ seg.

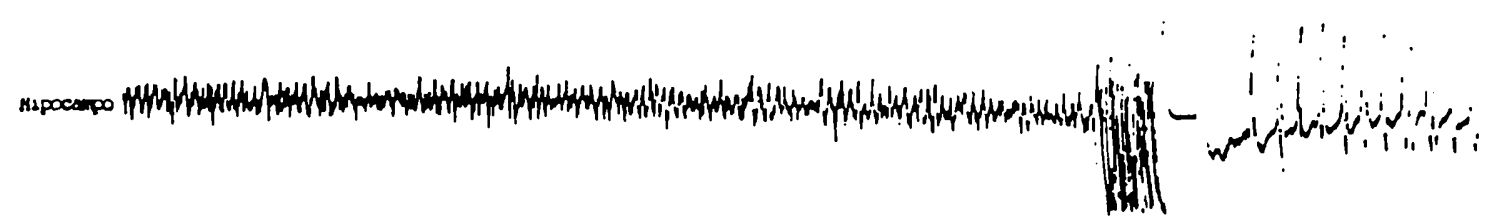

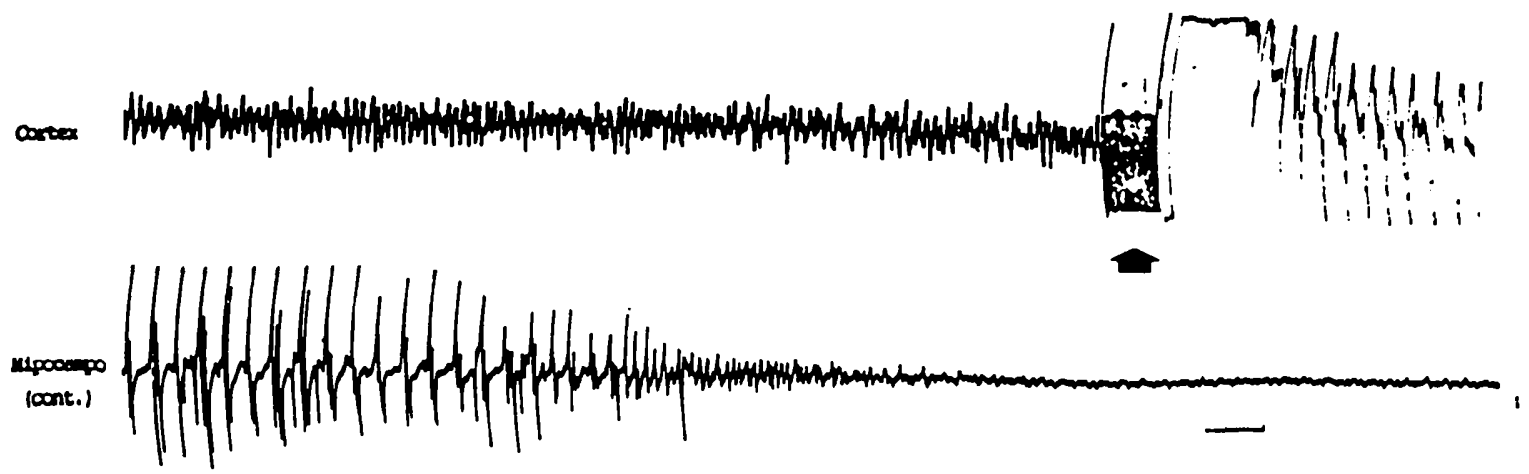

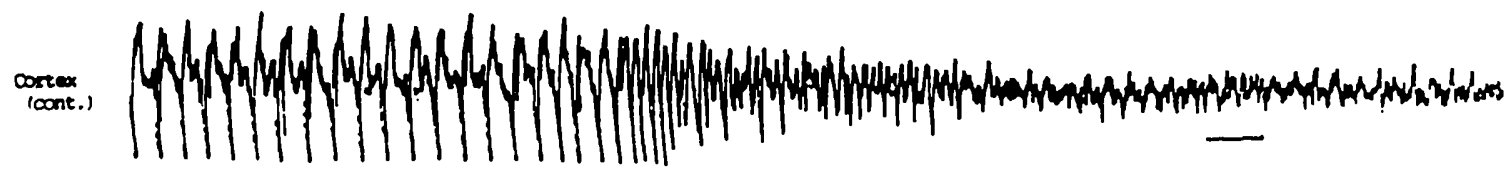

Fig. 4 - Observa-se, aposs o artefato de estimulo (seta), uma pós-descarga hipocampal e neocortical típica seguida de uma depressðo do amplitude do registro, mais evidente do hipocampo. Calibraçăo $=50$ $\mu \nabla / 1$ seg. 
Duração da atividade

epileptica eletrogräfica

(seg/minuto)

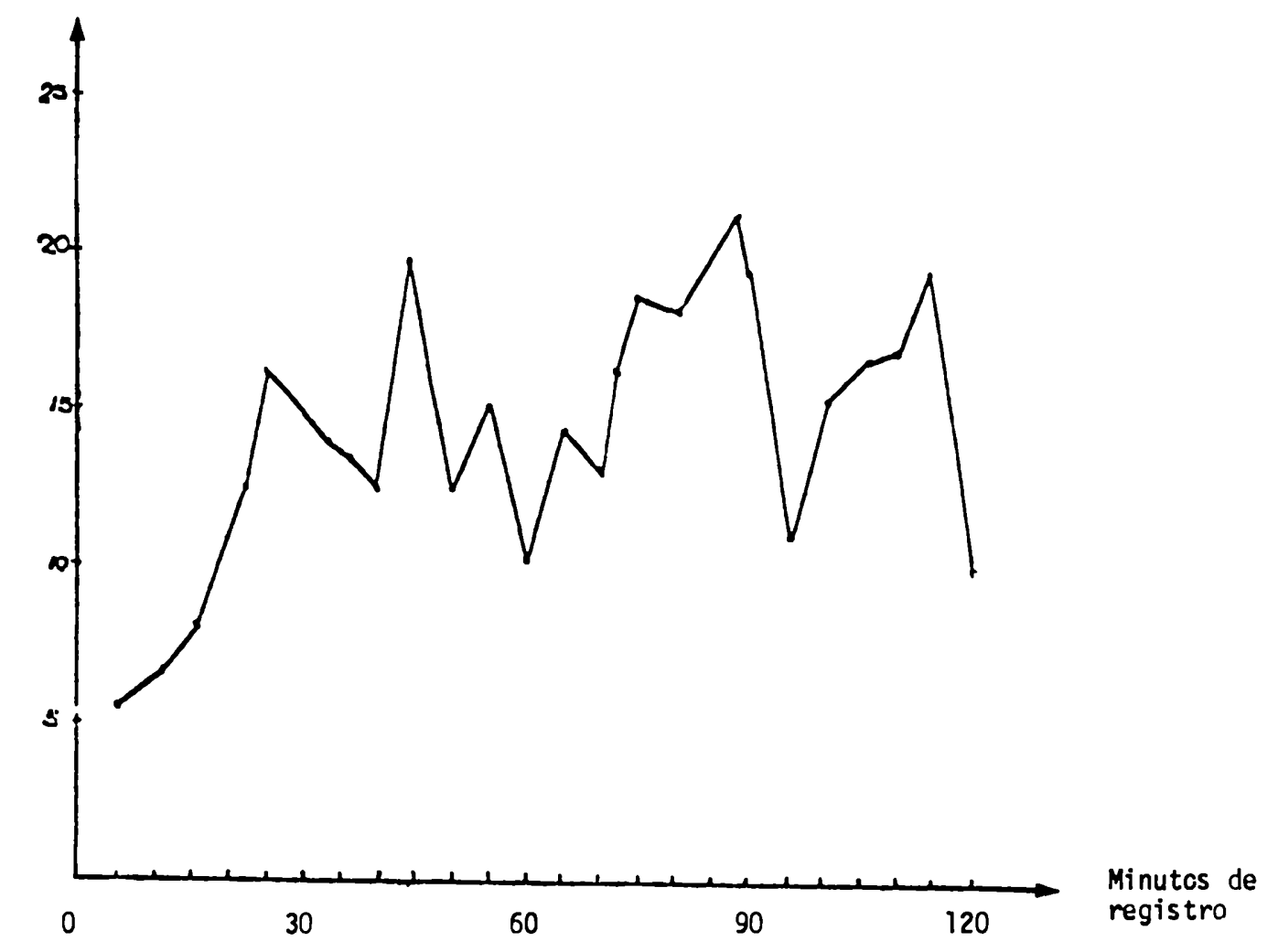

Grdfico 1 Duraço das pós-descargas durante 120 minutos de estimulą̧̃o. Observar que apos o s0" minuto a atividade permaneceu constantemente acima de 10 seg/min.

Duração (em porcentagem) da atividade epiléptica

eletrogrāfica

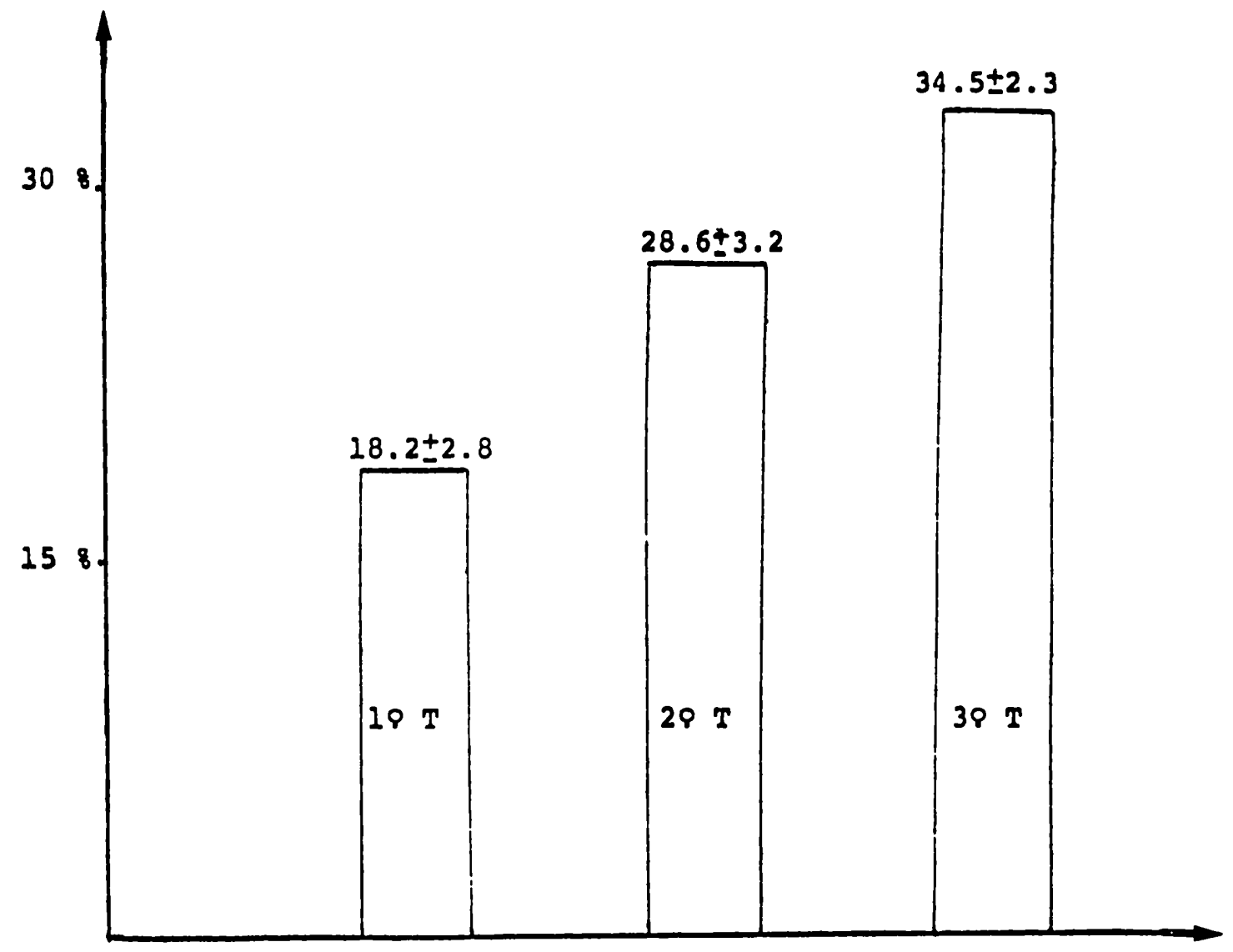

Grafico \&-Aumento da duraçao da atividade epiléptica eletrografica em sessōes repetidas de estimulação. 
Com relaçåo à duraçåo das pós-descargas durante os 120 minutos de estimulação, pode-se observar no gráfico 1 , que nos primeiros 30 minutos de estimulação houve aumento progressivo das mesmas, após o que a duração das pós-descargas permaneceu praticamente constante, durando aproximadamente $25 \%$ do tempo de registro.

Três animais foram ulteriormente retestados por mais duas vezes, no 149 e no 210 dia após a cirurgia, observando-se os resultados do gráfico 2 , onde se nota um aumento progressivo na duração da atividade epiléptica eletrográfica em sessões repetidas de estimulação.

\section{DISCUSSAO}

Pelos resultados apresentados podemos notar o estabelecimento definido do fenômeno "kindling" em prazo relativamente curto (30 minutos), em relação aos métodos já clássicos, nos quais se faz, habitualmente, um estímulo diário, em aproximadamente 15 ou 20 dias (Goddard e col. 1969).

Kaada (1951) verificou que, embora a forma e frequência das pós-descargas pudessem variar consideravelmente em cada área, o tipo de pós-descargas iniciado em cada região era surpreendentemente uniforme de um experimento para outro, mesmo variando o estímulo aplicado, em duração, intensidade, frequência e duração do pulso, não chegando a influenciar significativamente a forma $e$ frequência das pós-descargas iniciadas em um dado ponto.

Esses resultados não foram confirmados no presente experimento, pois verificamos grande variação no tipo de pós-descargas iniciadas no hipocampo e no neocórtex. Os vários padrões de pós-descargas observados em nosso experimento são similares aos descritos em trabalhos anteriores por Delgado e Sevillano (1961) e Racine (1972a). Notamos também, variações na frequência e amplitude das espículas numa mesma pós-descarga. Verificamos, além disso, a possibilidade do aparecimento de descargas hipocampais em forma de pontaonda de 3 ciclos por segundo, com projeção simultânea para áreas neocorticais bilaterais semelhantes às descritas para a chamada epilepsia "centrencefálica", tipo "Petit Mal" (ausência simples) de pacientes epilépticos.

O fenômeno "kindling" em áreas límbicas pode ser visto como uma ilustração dos efeitos facilitatórios da atividade epiléptica no desencadeamento subsequente de crises motoras. Atualmente é bem estabelecido que o "kindling" também envolve processos inibitórios. Foi verificado, por exemplo, que o "kindling" é bloqueado por uma redução do intervalo inter-estímulos para menos que 20 minutos e, com estimulação em intervalos pequenos, poderia eventualmente falhar o desencadeamento de respostas motoras de animais com "kindling" já estabelecido. Além disso, a obtenção de "kindling" a partir de um outro lugar do cérebro poderia produzir a supressão de crises desencadeadas por estimulação subsequente da área previamente incendiada ("kindled") (Goddard e col, 1969).

Verificamos no presente trabalho, que o reteste dos animais após uma semana leva a um aumento da atividade epiléptica eletrográfica e comporta- 
mental. Wada (1976) sugere que este fenômeno se deve à manutenção temporal da modificação plástica decorrente da estimulação elétrica repetitiva. Sessões repetidas de estimulação elétrica intermitente, em dias ulteriores, produzem resultados ainda mais acentuados. Já foi demonstrado por Racine (1972b) que esse aumento não depende do número de estímulos aplicados, mas sim do número de pós-descargas desencadeadas com a sua aplicação.

O método aqui utilizado demonstra que é possivel obter, em ratos, em curto espaço de tempo de experimentação uma situação de status epilepticus prolongado. Os nossos animais apresentaram periodos de epilepsia eletrográfica e comportamental ininterruptos por vários minutos, que se repetiram sucessivamente, sem um retorno completo à atividade eletrográfica normal. $\mathrm{O}$ fenômeno se enquadra, portanto, dentro das concepções clássicas de status epilepticus, como sendo crises convulsivas ininterruptas ou recidivantes, sem recuperação funcional entre elas, ou segundo o "Dictionary of Epilepsy" da OMS, editado por H. Gastaut (1973), que define o status epilepticus como "um estado caracterizado por uma crise epiléptica suficientemente prolongada ou repetida em intervalos suficientemente breves, que produz uma condição não variável e duradoura".

\section{RESUMO}

Foi utilizada em nova espécie animal (ratos), uma modificação do método "kindling", introduzida por Taber e col. (1977) para obtenção de status epilepticus experimental.

Para isso foram implantados mediante cirurgia estereotáxica, eletrodos duplos, torcidos no hipocampo dorsal de 12 ratos machos albinos. Esses animais foram submetidos, após uma semana de pós-operatório, a 1 segundo de estimulação elétrica de baixa intensidade em forma intermitente, um estímulo por minuto durante 2 horas, desenvolvendo-se em prazo de 30 minutos um estado de epilepsia eletrográfica e comportamental duradoura. Vários padrões de descargas epilépticas eletrográficas foram observados bem como manifestações convulsivas tônico-clônicas.

Os animais que foram submetidos a novas sessões de estimulação após 7 e 14 dias mostraram aumento de atividade epiléptica demonstrando uma modificação plástica do hipocampo do rato submetido a estimulação elétrica a qual perdura no tempo.

O método permite a obtenção de "kindling" em tempo bem mais curto (horas), comparativamente às técnicas anteriormente descritas (dias), tornando-se um promissor modelo de epilepsia para testes de drogas anticonvulsivantes e para o estudo dos mecanismos fisiopatológicos e bioquímicos envolvidos na descarga epiléptica. 


\section{SUMMARY}

\section{Experimental status epilepticus: a modified kindling method in rats.}

Taber et al. (1977) introduced new model of epilepsy to obtain .experimental status epilepticus in mice, through a modification of the kindling method (Goddard et al. 1969). The aim of this paper is to report the effect of Taber's modification on a new animal specie (rats).

Bipolar, twisted steel electrodes were stereotaxically implanted into the CA1-CA3 regions of the dorsal hippocampus. After one week the animals received 2 h stimulation sessions, one stimulus per minute, during which a sustained electrographical and behavioral seizures were induced. Different patterns of electrographical discharges as well as tonic-clonic convulsions were observed.

The animals which were submitted to 3 stimulating sessions respectively 7, 14 and 21 days after surgery showed an increase in the epileptic activity. This has been interpreted as a plastic. neural modification of the hippocampus similar to that observed during learning and memory consolidation.

In comparison to other inducing kindling methods this one permits a more rapid elicitation of the phenomenon. For this reason this method will provide a good epilepsy model for the study of anticonvulsant drugs and basic mechanisms involved in the epileptic activity.

\section{REFERENCIAS}

1. DELGADO, J. M. R. \& SEVILLANO, M. - Evolution of repeated hippocampal seizure in the cat. Electroencephalog. Clin. Neurophysiol. 13:722, 1961.

2. GastaUT, H. - Dictionary of Epilepsy. Part 1. Definitions. World Health Organization, Geneva, 1973.

3. GODDARD, G. V. - Development of epileptic seizures through brain stimulation at low intensity. Nature 214:1020, 1967.

4. GODDARD, G. V.; McINTYRE, D. C. \& LEECH, C. K. - A permanent change in brain function resulting from daily electrical stimulation. Exp. Neurol. 25:295, 1969.

5. KAADA, B. R. - Somato-motor, autonomic and electrocorticographic responses to electrical stimulation of rhinencephalic and other structures on primate, cat and dog. Acta Physiol. Scand. 23: suppl 83, 1-285, 1951.

6. RACINE, R. J. - Modification of seizure activity by electrical stimulation: after discharges threshold. Electroenceph. Clin. Neurophysiol. 32:269, 1972.

7. RACINE, R. J. - Modification of seizure activity by electrical stimulation: motor seizure. Flectroenceph. Clin. Neurophysiol. 32:281, 1972b.

8. TABER, K. H.; McNAMARA, J. J. \& ZORNETZER, S. F. - Status epilepticus: a new rodent model. Electroenceph. Clin. Neurophysiol. 43:707, 1977.

9. WADA, J. A. \& SATO, M. - Generalized convulsive seizure induced by daily electrical stimulation of the amygdala cats. Neurology (Minneapolis) 24:565, 1974.

10. WADA, J. A.; OSAWA, T. \& MIZOGUCHI, T. - Recurrent spontaneous seizure state induced by prefrontal kindling in senegalese baboons (Papio-Papio) In Wada, J. A. (Ed.) - Kindling. Raven Press, New York, pags 173-202, 1976.

Dr. Esper A. Cavalheiro - Neurofisiologia, Depto. de Fisiologia - Escola Paulista de Medicina - Rua Botucatu 868, \&p andar - 04083 Sao Paulo, SP - Brasil. 